\title{
Surgical Outcome of Prosthetic Heart Valve Obstruction: Single Center Experience
}

\author{
Alaa Brik ${ }^{1 *}$, Abdel-Maged Salem ${ }^{1}$, Nader Talat ${ }^{2}$, Amira Shoukry $^{3}$ \\ ${ }^{1}$ Department of Cardiothoracic Surgery, Zagazig University Hospital, Zagazig, Egypt \\ ${ }^{2}$ Department of Cardiology, Zagazig University Hospital, Zagazig, Egypt \\ ${ }^{3}$ Department of Internal Medicine, Zagazig University Hospital, Zagazig, Egypt \\ Email: *alaabrik@yahoo.com
}

Received September 6, 2012; revised October 29, 2012; accepted November 8, 2012

\begin{abstract}
Objective: Valve thrombosis defined as any thrombus not caused by infection attached to or near an operated valve that occludes part of the blood flow path and interferes with valve function. Treatment modalities are thrombolytic therapy, surgical thrombectomy and debridement, or re-replacement. The aim of this study is to review our experience in surgical thrombectomy and debridement for obstructed mechanical valve prosthesis, and the impact of the procedure on the outcome in comparison to re-replacement; Methods: The study was conducted retrospectively between 1995 to 2012 in Department of Cardiothoracic surgery, cardiology and internal medicine, Zagazig University Hospital. 92 patients with stuck valve were divided into two groups. Group (A): 54 patients with re replacement and Group (B): 38 patients treated by thrombectomy and debridement; Results: 92 patients with stuck valves the mean age was $38.4( \pm 9.2), 68$ male and 85 female in both groups. The large numbers of cases were mitral valve $35(64.8 \%)$ patients in group A and $22(57.9 \%)$ patients in group B, aortic malfunction in group A was 11 (20.4\%)patients and $9(23.7 \%)$ cases in group B, double valve malfunction was rare represent 8 (14.8\%) and 7 (18.4\%)cases in group A and group B respectively; Conclusion: We conclude that thrombectomy and debridement for malfunctioning mechanical valve is a safe, easy and rapid procedure to perform in some cases to avoid the risk of re replacement.
\end{abstract}

Keywords: Malfunction Valve; Mechanical Valve; Thromboembolism

\section{Introduction}

Prosthetic valve obstruction is a life-threatening complication after valve replacement [1]. The etiology usually due to Obstructionwhich caused by thrombus and/or punnus formation. Valve thrombosis defined as any thrombus not caused by infection attached to or near an operated valve that occludes part of the blood flow path and interferes with valve function, or is sufficiently large to needs treatment [2]. The clinical presentation of mechanical prosthetic valve obstruction varies from mild symptoms, progressive heart failure, embolic manifestations, or hemodynamic compromise [3]. Treatment modalities are thrombolytic therapy, surgical thrombectomy, or replacement. The purpose of this study is to review our experience in surgical thrombectomy for obstructed mechanical valve prosthesis, and the efficacy and impact of the procedure on the outcome.

\section{Methods}

\subsection{Study Population and Data Collection}

Our report is retrospective study conducted between 1995

${ }^{*}$ Corresponding author. to 2012, in departments of cardiothoracic surgery, cardiology and internal medicine Zagazig University Hospital. 92 patients with stuck valve were divided into two groups according to the type of surgical treatment. Group (A): 54 patients treated with re-replacement and Group (B): 38 patients treated by surgical thrombectomy and debridement of punnus.

\subsection{Pre-Operative Assessment}

Accurate history taking and clinical evaluation were done to all patients. Clinical data suggestive of prosthetic valve obstruction where: inadequate anticoagulation, diminished or absent click, progressive heart failure, low cardiac output, and pulmonary edema Immediate echocardiography was done to confirm the diagnosis as soon as prosthetic valve obstruction was suspected. Transthoracic echocardiography was sufficient for diagnosis. Transeosophageal echocardiography was necessary in 15 patients. Assessment of valve movement, valve area, transvalvular gradient, presence of thrombi, punnus or both, pulmonary artery pressure were carefully measure. We did not use cinefluoroscopy for diagnosis since clinical and echocardiographic data were sufficient and we tried not to lose 
time for additional investigations.

\subsection{Surgical Technique}

Enrolled patients in this study undergo prosthetic valve thrombectomy thrombolytic therapy was attempted in some cases which excluded from our study because the patients which not respond for thrombolytic therapy were submitted to surgical treatment and we wanted to compare the two main surgical options, 92 patients were submeted for surgery.

We used femoral bypass in one patient due to bleeding during sternotomy. Antegrade warm blood cardioplegia was used for myocardial protection in all patients. Mitral valve was approached through the left atrium or transsepal approach and aortic valve through standard aortotomy.

Mitral valve prosthesis was assessed, exposed by traction sutures and any thrombi or punnus were removed (Figure 1). We used fine nerve hook to clean the ventricular surface of the valve. We occasionally perform exploratory aortoromy to ensure freedom of the ventricular surface especially when we decided irrigation of the left ventricle. Rotation of the valve in some cases for insuring complete removal of thrombi, then we test the valve to assessed the valve mobility and function (Figure 2).

Replacement was decided in cases associated with valve dehiscence or inadequate removal of the thrombus/pannus which affect the valve movement.

Postoperatively, patients were admitted to ICU, and gradually weaned from mechanical ventilation and inotropic support. They were discharged from hospital on oral anticoagulant with target INR 3 - 3.5 and on aspirin $75 \mathrm{mg}$ POD.

All enrolled patients were followed up for 6 months in the form of visiting in outpatient clinic every 2 weeks, TTE examination within the first month postoperatively.

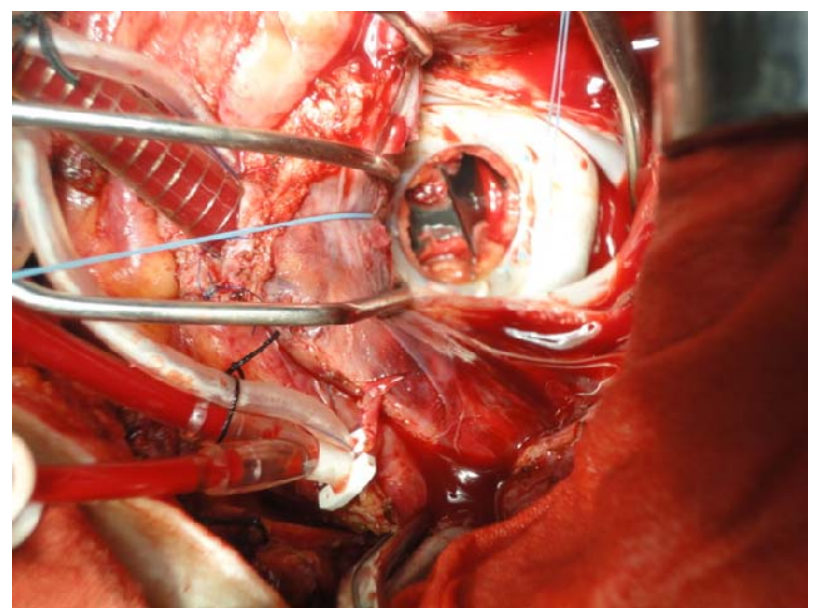

Figure 1. Show thrombus obstructed the mechanical valve and the traction sutures for good projection.

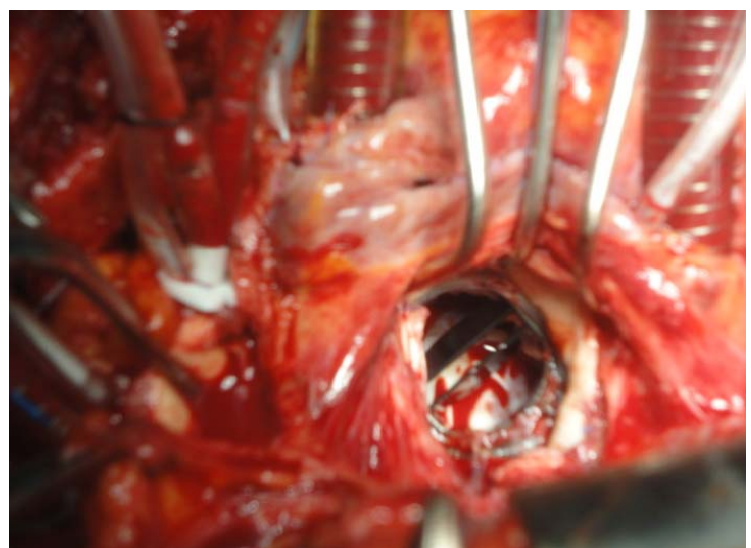

Figure 2. Shows the mechanical bileaflet valve after removal of the thrombus.

\subsection{Statistical Analysis}

Results were analyzed using SPSS version 15.0 (Statistical Package for Social Science, SPSS Inc., Chicago, IL, USA). Continuous variables, such as age were expressed as the mean \pm S.D. and compared by unpaired t-test. Categorical variables were expressed by number $(n)$ and frequencies $(\%)$. The $\chi^{2}$-test was used to compare the proportions. A P value of $<0.05$ was considered statisticcally significant.

\section{Results}

Collection of the data since 1995 for all patients presented by obstructed mechanical valve prosthesis. The patient's diagnosis by clinical data and transthoracic echocardiography (TTE) and in 15 cases transesophegeal echocardiography (TEE) was needed. 92 patients with stuck valve were divided into two groups. Group (A): 54 patients with replacement and Group (B): 38 patients treated by debridement and removal of thrombus or punnus.

The mean age in group A was $36.4 \pm 8.2$ and in group B was $42.3 \pm 9.8,39(42.3 \%)$ male and $53(57.6 \%)$ female in both groups The large numbers of cases were mitral valve 35 (64.8\%) patients in group A and 22 (57.9\%) patients in group B, aortic valve obstruction in group A 11 $(20.4 \%)$ patients and $9(23.7 \%)$ cases in group B, double valves obstruction was rare represent $8(14.8 \%)$ and 7 (18.4\%) cases in group A and group B 18.4\% respectively Table $\mathbf{1}$.

The types of mechanical valves were $49(90.7 \%)$ cases with bileaflets valves in group A and all cases in group B, while ball and cage and tilting disc valves were zero percent in group B and 5 cases in group A, 2 ball and cage and 3 tilting disc valve.

The INR (international randomized ratio) is under therapeutic range in the majority of patients INR less than 2 in $44(81.5 \%)$ and $30(78.9 \%)$ in group A and B respectively. 
Embolic manifestation was found in $3(5.6 \%)$ patients in group A and not detected in group B. pregnant women with malfunctioning valve were 5 cases $(9.25 \%)$ in group A and 2 cases $(5.26 \%)$ in Group B all of the mitral position.

The mortality was 3 cases $(5.6 \%)$ in group A, one case due to massive operative bleeding during re sternotomy and the two cases died in post-operative period one case due to multiorgans failure and the other case by medistinitis. Post-operative embolic manifestation and paravalvular leaks in $2(3.7 \%)$ cases in group $\mathrm{A}$ and no cases in group B. Re-thrombosis or new punnus formation were noted in $4(7.4 \%)$ cases in group $\mathrm{A}$ in outpatient clinic follow up all of them were females, one of them was pregnant and no recorded cases in group B. Other complications like chronic chest pain, arrhythmia and bleeding occurred in both groups with no significant differences Table 2.

\section{Discussion}

Until now thromboembolism of the mechanical valves remains one of the most important complications in patients undergoing valve replacement Since 1950, a number of patient-related factors canincrease the thromboembolic risk, irrespective of the devicetypes and the location of the valves. Prosthetic valve thrombosis has reportedly occurred with both mechanical and bio prosthetic heart valve [4] in our study we found the majority of the patients were in mitral position and with bileaflet mechanical heart valves, The term "thrombosis" includes several pathological findings ranging from primary thrombosis,

Table 1. Patient's characteristics.

\begin{tabular}{|c|c|c|c|c|c|}
\hline & \multicolumn{2}{|c|}{ Group A (54) } & \multicolumn{2}{|c|}{ Group B (38) } & $P$ value \\
\hline Age & \multicolumn{2}{|c|}{$36.4( \pm 8.2)$} & \multicolumn{2}{|c|}{$42.3( \pm 9.8)$} & 0.002 \\
\hline \multicolumn{6}{|l|}{ Sex } \\
\hline Male & 21 & $38.9 \%$ & 18 & $47.4 \%$ & 0.41 \\
\hline Female & 33 & $61.1 \%$ & 20 & $52.6 \%$ & \\
\hline \multicolumn{6}{|l|}{ Obstructed valve } \\
\hline Mitral & 35 & $64.8 \%$ & 22 & $57.9 \%$ & 0.58 \\
\hline Aorta & 11 & $20.4 \%$ & 9 & $23.7 \%$ & 0.81 \\
\hline Double valve & 8 & $14.8 \%$ & 7 & $18.4 \%$ & 0.28 \\
\hline \multicolumn{6}{|c|}{ Type of mechanical valve } \\
\hline Ball and cage valve & 2 & $3.7 \%$ & 0 & & 0.5 \\
\hline Tilting disc valve & 3 & $5.6 \%$ & 0 & & 0.26 \\
\hline Bileaflet valve & 49 & $90.7 \%$ & $38 \%$ & $100 \%$ & 0.07 \\
\hline INR (Less than 2) & 44 & $81.5 \%$ & 30 & $78.9 \%$ & 0.76 \\
\hline Embolic manifestation & 3 & $5.6 \%$ & 0 & & 0.26 \\
\hline Atrial fibrillation & 22 & $40.7 \%$ & 12 & $31.5 \%$ & 0.55 \\
\hline Diabetes mellitus & 6 & $11.1 \%$ & 4 & $10.5 \%$ & 0.8 \\
\hline Pregnancy & 5 & $9.25 \%$ & 2 & $5.26 \%$ & 0.69 \\
\hline Renal dysfunction & 1 & $1.85 \%$ & 0 & & 0.85 \\
\hline Liver dysfunction & 3 & $5.6 \%$ & 1 & $2.6 \%$ & 0.87 \\
\hline
\end{tabular}

INR: international randomized ratio.

Table 2. Postoperative complications.

\begin{tabular}{|c|c|c|c|c|c|}
\hline \multirow[b]{2}{*}{ Re-thrombosis or punnus } & \multicolumn{2}{|c|}{ Group A (54) } & \multicolumn{2}{|c|}{ Group B (38) } & \multirow{2}{*}{$\frac{P \text { value }}{0.13}$} \\
\hline & 4 & $7.4 \%$ & 0 & & \\
\hline Embolic manifestation & 2 & $3.7 \%$ & 0 & & 0.5 \\
\hline Mediastinitis & 1 & $1.85 \%$ & 0 & & 0.85 \\
\hline Paravalvular leak & 2 & $3.7 \%$ & 0 & & 0.5 \\
\hline Chronic chest pain & 6 & $11.1 \%$ & 2 & $5.26 \%$ & 0.46 \\
\hline Arrhythmia & 12 & $22.2 \%$ & 6 & $15.8 \%$ & 0.44 \\
\hline Bleeding & 4 & $7.4 \%$ & 3 & $7.9 \%$ & 0.75 \\
\hline
\end{tabular}


pannus formation and secondary thrombosis [5].

The thrombogenic potential is believed to be highest for the older-generation caged-ball or caged-disc valves, while single tilting-disc valves have been reported to have a lower thrombogenicity than caged-ballprostheses. It is generally believed that the latest generation of bileaflet prostheses have the lowest risk of thromboembolism [6]. Overall, the rate of embolic events is reported to range from 0.6 to $6.5 \mathrm{pt}-\mathrm{yr}$ with bileaflet prostheses [7], and from $1.5 \%$ to $4.7 \%$ per pt-yr for single tilting-discdevices depending on valve design, structure, location and patient compliance with oral anticoagulation $[7,8]$. About $62 \%$ of enrolled patients, as well as, all patients with recurrent thrombosis or punnus formation after thrombectomy or debridement were females. This asserts the assumption that female sex is a risk factor for acute thrombotic occlusion [8]. Inadequate anticoagulation was the cardinal cause of prosthetic valve thrombosis in our study particularly due to in attentive follow-up and shift from oral anticoagulant to subcutaneous heparin during pregnancy, emphasizing the need for both patient and physician oriented education [9]. The presentation of patients with valve obstruction ranges from progressive heart failure to cardiogenic shock. Any new or worsening symptom or an embolic event in a patient with prosthetic valve should prompt thorough investigation to rule out valve thrombosis. In accordance with literature [10] noting that shorter duration of symptoms is typical for thrombi, while longer duration signifies usually the presence of pannus.

Signs of thrombosis are an increase in the transprosthetic pressure gradient $>2 \times$ normal values. Valve obstruction without regurgitation was the leading hemodynamic Doppler echocardiographic finding in our study. The majority of cases in this work had combined obstruction and regurgitation. TEE was necessary in 15 patients as it facilitates better visualization of thrombi, mechanism of obstruction, and abnormal prosthetic leaflet or disc motion [11].

Thrombolysis, thrombectomy or prosthetic replacement is current available treatment options for prosthetic valve thrombosis [12]. Low-output states, cardiogenic shock, as well as absolute contraindications limit thrombolysis usage [13]. Previous studies sheId light upon a high risk of increased embolic insults in NYHA class I or II with thrombolysis and relatively higher morbidity and mortality with left-sided prosthetic valve thrombosis $[14$, $15]$.

In previous report re-do replacement of thrombosedprosthesis may imply long cross-clamping and CPB times. It should be restricted to cases of extensive pannus or if mechanical damage is found [16] and this agree with our concept that we should avoid re-replacement if possible to avoid prolonged operation with long cross clamp. When prosthetic valve thrombosis is related to an inadequate level of anticoagulation, thrombectomy which is easy, safe, and fast could be an excellent approach when all parts of thrombus are removed [13].

A previous met analysis [17] had showed that recurrent thrombosis rate does not differ significantly between thrombectomy and valve re-replacement, this was encouraging to us in the decision making process and this findingbroadly appeared in our study when all cases of rethrombosis were in Group A by (7.4\%).

We had one case died intraoperatively from uncontrolled bleeding and the other two cases died in early postoperative period. All of them were operated in emergency situation with cardiogenic shock and hemodynamic instability preoperatively which is a surgical mortality risk factor as highlighted in previous studies $[15,18,19]$.

We had 4 cases of re-thrombosis in cases treated by rereplacement One of them caused by shift from oral anticoagulant to heparin during pregnancy and all of them had Atrial Fibrillation (AF). So our strategy is to continue oral anticoagulant all over the period of gestation as previous reports, Warfarin was more effective than heparin in preventing thromboembolism in the mothers, and it did not show a significant impact on the babies [20].

Limitation of this study is its retrospective nature and the lack of using radiofrequency ablation with thrombectomy to alleviate AF, to improving the outcome and decreasing re-thrombosis incidence.

\section{Conclusion}

We conclude that thrombectomy and debridement for obstracted mechanical valveoccurred by thrombosis or punnus formation is a safe, easy, fast and favorable outcome particularly when all parts of the thrombus or punnus are removed leaving mobile and functioning valve to avoid the risk of re replacement.

\section{REFERENCES}

[1] D. Horstkotte and D. Burckhardt, "Prosthetic Valve Thrombosis," Journal of Heart Valve Disease, Vol. 4, No. 2, 1995, pp. 141-153.

[2] C. W. Akins, D. C. Miller, M. I. Turina, N. T. Kouchoukos, E. H. Blackstone, G. L. Grunkemeier, J. J. M. Takkenberg, T. E. David, E. G. Butchart, D. H. Adams, D. M. Shahian, S. Hagl, J. E. Mayer and B. W. Lytle, "Guidelines for Reporting Mortality and Morbidity after Cardiac Valve Interventions," The Journal of Thoracic and Cardiovascular Surgery, Vol. 135, 2008, pp. 732-738. doi:10.1016/j.jtcvs.2007.12.002

[3] P. Buttard, E. Bonnefoy, P. Chevalier, P. B. Marcaz, J. Robin, J. F. Obadia, P. Touboul and G. Kirkorian, "Mechanical Cardiac Valve Thrombosis in Patients in Critical Hemodynamic Compromise," European Journal CardioThoracic Surgery, Vol. 11, No. 4, 1997, pp. 710-713. 


\section{doi:10.1016/S1010-7940(96)01133-5}

[4] M. T. Hagley, A. Lopez-Candales, K. J. Phillips, B. B. Daily and N. T. Kouchoukos, "Thombosis of Mitral Valve Bioprostheses in Patients Requiring Circulatory Assistance," The Annals of Thoracic Surgery, Vol. 60, No. 6, 1995, pp. 1814-1816. doi:10.1016/0003-4975(95)00627-3

[5] A. Ranzulli, F. Onorati, M. DeFeo, N. Vitale, S. Esposito, L. Agozzino, G. Santarpino, P. Mastroroberto, A. R. Marchese, L. D. Luca, M. Scardone and M. Cotrufo, "Mechanical Valve Thrombosis: A Tailored Approach for a Multiplex Disease," Journal of Heart Valve Disease, Vol. 13, No. 1, 2003, pp. S37-S42.

[6] P. D. Stein, H. I. Bussey, J. E. Dalen and A. G. G. Turpie, "Antithrombotic Therapy in Patients with Mechanical and Biological Prosthetic Heart Valves," Chest, Vol. 119, No. 1, 2001, pp. 220s-226s. doi:10.1378/chest.119.1 suppl.220S

[7] D. Horstkotte, H. Schulte, W. Bircks and B. E. Strauer, "Lower Intensity Anticoagulation Therapy Results in Lower Complication Rates with the St. Jude Medical Prostheses," The Journal of Thoracic and Cardiovascular Surgery, Vol. 107, No. 4, 1994, pp. 1136-1145.

[8] A. C. Fiore, H. B. Barner, M. T. Swartz, L. R. McBride, A. J. Labovitz, K. J. Vaca, J. St. Vrain, G. L. Grunkemeier and G. C. Kaiser, "Mitral Valve Replacement: Randomized Trial of St. Jude and Medtronic Hall prostheses," The Annals of Thoracic Surgery, Vol. 66, No. 3, 1998, pp. 707-713. doi:10.1016/S0003-4975(98)00670-5

[9] S. Robert, A. Raymond, P. Jobn, J. Micheal, W. William and A. Eugene, "Prosthetic Heart Valve Thrombosis: An Overview," Wisconsin Medical Journal, Vol. 101, No. 7, 2002, pp. 67-69.

[10] B. Kogon, P. H. Kirshbom, J. M. Forbess and K. R. Kanteret, "Thrombolytic Therapy for Progressive Valve Thrombosis in Children: Two Case Reports and Review of Literature," The Journal of Thoracic and Cardiovascular Surgery, Vol. 127, No. 5, 2004, pp. 1519-1522. doi:10.1016/i.jtcvs.2003.11.026

[11] J. Barbetseas, S. F. Nagueh, C. Pitsavos, P. K. Toutouzas, M. A. Quiñones and W. A. Zoghbi, "Differentiating Thombus from Pannus Formation in Obstructed Mechanical Prosthetic Valves: An Evaluation of Clinical, Transthoracic and Transesophageal Echocardiographic Parameters," Journal of the American College of Cardiology, Vol. 32, No. 5, 1998, pp. 1410-1417. doi:10.1016/S0735-1097(98)00385-4
[12] A. A. Aroussi, I. M. Sami and A. Leguerrier, "The Blower: A Useful Tool to Complete Thrombectomy of the Mechanical Thrombotic Valve," The Annals of Thoracic Surgery, Vol. 81, No. 5, 2006, pp. 1911-1912. doi:10.1016/j.athoracsur.2005.02.070

[13] M. Lengyel, V. Fuster, M. Keltai, R. Roudaut, H. D. Schulte, J. B. Seward, J. H. Chesebro and A. G. Turpie, "Guidelines for Management of Left-Sided Prosthetic Valve Thrombosis: A Role for Thrombolytic Therapy. Consensus Conference on Prosthetic Valve Thrombosis," Journal of the American College of Cardiology, Vol. 30, No. 6, 1997, pp. 1521-1526. doi:10.1016/S0735-1097(97)00345-8

[14] H. Silber, S. S. Khan, J. M. Matloff, A. Chaux, M. DeRobertis and R. Gray, "The St. Jude Valve Thrombolysis as the First Line of Therapy for Cardiac Valve Thrombosis," Circulation, Vol. 87, No. 1, 1993, pp. 30-37. doi:10.1161/01.CIR.87.1.30

[15] G. J. Kontos, H. V. Schaff, T. A. Orszulak, F. J. Puga, J. R. Pluth and G. K. Danielson, "Thrombotic Obstruction of Disc Valves: Clinical Recognition and Surgical Management," The Annals of Thoracic Surgery, Vol. 48, No. 1, 1989, pp. 60-65. doi:10.1016/0003-4975(89)90177-X

[16] P. Venugopal, U. Kaul, K. S. Lyer, I. M. Rao, A. Balram, B. Das, A. Sampathkumar, S. Mukherjee, M. Rajani and H. S. Wasir, "Fate of thrombectomizedbjor Shiley Valves: A Long-Term Cinefluoroscopic, Chocardiographic and Hemodynamic Evaluation," The Journal of Thoracic and Cardiovascular Surgery, Vol. 91, No. 2, 1986, pp. 168-173.

[17] D. Hering, C. Piper and D. Horstkotte, "Management of Prosthetic Valve Thrombosis," European Heart Journal, Vol. 17, 2001, pp. 646-650.

[18] N. Vitale, A. Renzulli, F. Cerasuolo, A. Caruso, M. Festa, L. de Luca and M. Cotrufo, "Prosthetic Valve Obstruction: Thrombolysis versus Operation," The Annals of Thoracic Surgery, Vol. 57, No. 2, 1994, pp. 365-370. doi:10.1016/0003-4975(94)90998-9

[19] E. Deviri, P. Sareli, T. Wisenbaugh and S. L. Cronje, "Obstruction of Mechanical Heart Valve Prosthesis: Clinical Aspects and Surgical Management," Journal of the American College of Cardiology, Vol. 17, No. 3, 1991, pp. 646-650. doi:10.1016/S0735-1097(10)80178-0

[20] Z. A. Ashour, H. A. Shawky and M. H. Hussein, "Outcome of Pregnancy in Women with Mechanical Valves," Texas Heart Institute Journal, Vol. 27, No. 3, 2000, pp. 240245. 